\title{
Seasonal thermal neutron flux variations at high altitude
}

\author{
Yu.V. Stenkin ${ }^{1,2}$, V.V. Alekseenko ${ }^{1}$, Z.Y. Cai ${ }^{3}$, Z. Cao ${ }^{4}$,C. Cattaneo ${ }^{5}$, E. Giroletti ${ }^{5,6}$,
} S.W. Cui ${ }^{3 *}$, X.W. Guo ${ }^{3}$, D.M. Gromushkin ${ }^{2}$, H.H. He ${ }^{4}$, Y. Liu ${ }^{7}$, X.H. Ma ${ }^{4}$, O.B. Shchegolev $^{1}$, V.I.Stepanov ${ }^{1}$, A.L. Tsyabuk ${ }^{1}$, P. Vallania, ${ }^{8,9}$, C. Vigorito ${ }^{9,10}$ and J. Zhao $^{4}$

\footnotetext{
${ }^{1}$ Institute for nuclear Research, Russian Academy of Sciences, Moscow, Russia

${ }^{2}$ National Research Nuclear University MEPhI (Moscow Engineering Physics Institute), Moscow, Russia

3 The College of Physics Science and Information Engineering, Hebei Normal University, Shijiazhuang, China

${ }^{4}$ Key Laboratory of Particle Astrophysics, Institute of High energy Physics, Chinese Academy of Sciences, Beijing, China

${ }^{5}$ Dip. Fisica, Università Studi di Pavia, Pavia, Italy

${ }^{6}$ Istituto Nazionale di Fisica Nucleare, Pavia, Italy

${ }^{7}$ The School of Physics, Shandong University, Jinan, China

${ }^{8}$ Osservatorio Astrofisico di Torino dell'Istituto Nazionale di Astrofisica, Torino, Italy

${ }^{9}$ Istituto Nazionale di Fisica Nucleare, Torino, Italy

${ }^{10}$ Dipartimento di Fisica dell’Università di Torino, Torino, Italy
}

\begin{abstract}
The PRISMA project developed for cosmic ray study above $30 \mathrm{TeV}$ is now realized as 2 prototypes at different altitudes. Special en-detectors are used for both electron and neutron components recording. An array of 4 such detectors is running in Yang Ba Jing (Tibet, China) at altitude $4300 \mathrm{~m}$ a. s. l. since January 2013. To be sure that thermal neutron background is stable we also study its variations. Here we present the results of this study, namely seasonal thermal neutron flux variations at high altitude.
\end{abstract}

The 34th International Cosmic Ray Conference

30 July- 6 August, 2015

The Hague, The Netherlands

\footnotetext{
${ }^{*}$ Speaker
} 


\section{Introduction}

It is well known that radioactive gas ${ }^{222} \mathrm{Rn}$ originated from the radioactive chain of ${ }^{238} \mathrm{U}$ in soil and construction materials as inert gas can penetrate indoor environments and accumulate in basements and underground Laboratories. Its concentration in air can be rather high and it is strongly sensitive to site geology and many geophysical and microclimatic processes such as local seismic activity, air pressure and wind, tides and so on. A lot of work has been done to measure and keep under control the environmental radon concentration. Unfortunately, air radon concentration measurements are hampered by very big fluctuations due to air movement, ventilation, air humidity, etc. On the other hand, radon and its daughter nuclides are a -active and therefore there is a neutron flux in equilibrium with radon in the Earth's crust due to nuclear $(\alpha, n)$-reactions (not in air where no suitable targets for $(\alpha, n)$-reactions exist). These reactions produce fast neutrons with energy of a few $\mathrm{MeV}$, that are afterwards moderated down to thermal and epithermal energies covering several meters underground. The radon diffusion length in soil strongly depends on the site geology, ground water level, etc., a reasonable expectation being of the order of tens of meters. Most of neutrons are captured by surrounding nuclei, but some of them can escape absorption and come to air. Therefore, it is possible to monitor the radon concentration in soil by measuring the thermal neutron flux in air. This method developed by us [1,2] has many advantages, mainly the fact that it is not sensitive to air drought, ventilation, humidity, etc., but only to the radon concentration underground (in soil, rock, concrete, etc.). More precisely, the neutron yield is proportional to the radon density flux through soil, rock, concrete or other porous materials close to the detector.

\section{The experimental setup}

The en-detectors [3] were originally developed by us for cosmic ray Extensive Air Showers (EAS) study. The en-detector is sensitive to thermal and epithermal neutrons by means of a thin layer of inorganic scintillator $\mathrm{ZnS}(\mathrm{Ag})$ alloyed with ${ }^{6} \mathrm{LiF}$. ${ }^{6} \mathrm{Li}$ has a high cross section for the (n, a )-reaction: ${ }^{6} \mathrm{Li}+\mathrm{n} \rightarrow{ }^{3} \mathrm{H}+\mathrm{a}+4.78 \mathrm{MeV}$. Heavy charged particles ${ }^{3} \mathrm{H}$ and a produce pointlike ionization with the emission of $\sim 160000$ light photons in the $\mathrm{ZnS}(\mathrm{Ag})$ scintillator. Light is collected by a 5-inch photomultiplier tube FEU-200 obtaining a signal of about 100 photoelectrons from the PMT photocathode. A significant feature of this scintillator is the existence of several time constants (from 40 ns to hours) and its sensitivity to particle velocity: slowly moving heavy particles such as a or ${ }^{3} \mathrm{H}$ excite more efficiently slow components in the detector pulse shape. The scintillator, with a surface of $0.35 \mathrm{~m}^{2}$, and the PMT are located inside a black plastic tank of $200 \mathrm{l}$ volume used as detector housing. Data acquisition includes a full pulse shape digitizer with a flash analog-to-digital converter (FADC) installed in a PCI slot of the On-Line PC. The data of each detector are collected and stored every 5 minutes.

Due to the very thin scintillator layer (only $30 \mathrm{mg} / \mathrm{cm}^{2}$ ) charged particles (electrons, muons, etc.) lose only $\sim 60 \mathrm{keV}$ and their signals are below the FADC threshold set at $150 \mathrm{keV}$. Only neutron captures and synchronous passages of several charged particles can be detected, and this results in a very low en-detectors counting rate. Two trigger systems of the array have been implemented: 1) the coincidence of any 2 out of 4 detectors for EASs and 2) any hit of any detector with a conversion factor reduced by a factor of 16 to decrease the dead time. For variations study we use the second one with a counting rate of $\sim 2 \mathrm{~Hz}$. The scintillator efficiency of our scintillator for thermal neutron detection is $\sim 20 \%$. Using the detector pulse shape analysis we can identify three signal types: "neutrons" produced mostly by thermal neutron captures by target nuclei ${ }^{6} \mathrm{Li}$, "charged" caused by multiple charged relativistic particles passage and "very slow" due to electromagnetic noise. The signal separation is possible due to different charge collection times in case of passage of charged relativistic particles and of slowly moving alpha-particles and ${ }^{3} \mathrm{H}$ emitted after the neutron capture by ${ }^{6} \mathrm{Li}$ nuclei. All these signal types are 
counted and stored separately. The ratio of the neutron counting rate to the charged counting rate is $\sim 0.07$.

In order to see whether thermal neutrons generated by the radon concentration are indeed detectable at high altitude, we have built an array, so called PRISMA-YBJ[4], made by four endetectors at Yangbajing, Tibet, China, $4300 \mathrm{~m}$ a.s.l.. The neutron detector array is located near the center of the RPC array of the ARGO-YBJ experiment [5]. Three of the neutron detectors are at the vertices of an equilateral triangle with sides of $5 \mathrm{~m}$, and the other one is in the center. The results presented here have been obtained after late August, 2013.

\section{Results}

Seasonal variations of the en-detector counting rate are shown in Fig. 1 for pulses selected as "neutrons" (black line) and as "charged" (red line) with 1-week smoothing for the period from 30.08.2013 till 03.12.2014. For the same period, data for the "charged" channel are shown in Fig. 2 without any correction as 5-min series (light gray) and as 30-day-smoothed red line. Seasonal variations are evident in both channels. The variations in the "charged" channel with 1-week (Fig. 1) and particularly 1-month smoothing (Fig. 2) are almost sinusoidal while those for "neutrons" are affected by air pressure, temperature and presence of precipitations, since water is a good neutron moderator and absorber. It can be seen that the fall of the neutron flux intensity (Fig. 1) coincides with the beginning of the rain period in summer (Fig. 3). This coincidence occurs for both types of signals while the maxima are not coincident but both occur during the dry season. The $10 \%$ seasonal wave of air radon measured through our "charged" channel corresponds to an amplitude of $\sim 770 \mathrm{~Bq} / \mathrm{m}^{3}$ in the dry season.

The radon concentration in air measured through the Lucas cells (Fig. 4) is affected by many random parameters as wind, ventilation, etc. and do not show a clear seasonal effect. On the contrary, underground radon measurements are more stable and results shown in [6-8] confirm the existence of the seasonal effect on underground radon. Note that the amplitude of a seasonal wave in soil radon measured at Stromboli [6] was found to be of $\sim 2000 \mathrm{~Bq} / \mathrm{m}^{3}$ (or $\sim 50 \%$ ) with a maximum coincident with the driest season.

The smooth behavior of our "charged" data could be explained by the recording difference between Lucas cells and en-detectors: the "charged" channel is not sensitive directly to radon alpha decays but to cascades of gammas from beta-decays of radon daughters (mostly ${ }^{214} \mathrm{Bi}$ and ${ }^{214} \mathrm{~Pb}$ ) close to the detector. Heavy metals like these are highly ionized and can actively attach to charged aerosols or dust that can be moved by wind to long distances. Even inside the ARGOYBJ hall, they can be deposited on the detector surface, made of plastic and therefore able to attract electrostatically the dust. Clearly, this process depends strongly on weather conditions like wind, rainfalls and air humidity. During the dry season this pollutants transport is greater while it is smaller during the rainy season. Note that the radon meter has an air filter on its inlet pipe making it not sensitive to dust and aerosols. This justifies the difference found between the "charged" and Lucas cells data.

The results show that the concentrations of underground radon and of thermal neutrons in equilibrium with it are higher during the dry period and that the difference between dry and wet periods could be of about $20 \%$ in radon-due thermal neutrons, while direct radon measurements in air gave between $30 \%$ and $40 \%$ for the seasonal variations in soil [7]. Our "charged" counts due to the decays of radon daughters carried by dust shows a smooth seasonal wave of $10 \%$ amplitude. We should note here that these counts are produced not only by radon decays but also by other processes, such as small Extensive Air Showers (EAS), interactions of cosmic rays just above the detector and even PMTs noise. It is probably for this reason that we found an amplitude of the "charged" counts seasonal wave smaller than that obtained with direct radon measurements. 


\section{Conclusions}

New results were obtained using a new nuclear physics method based on continuous measurement of: 1) ${ }^{222} \mathrm{Rn}$ concentration in air; 2) ${ }^{222} \mathrm{Rn}$ daughters in dust (aerosols) and 3) ${ }^{222} \mathrm{Rn}$ flux density in soil through the detection of thermal neutrons produced in $(a, n)$-reactions underground by the alpha particles originated from the radon decay chain. The existence of periodical seasonal variations has been confirmed for the first time at high altitude. Moreover, the existence of the seasonal effect for radon and thermal neutrons in anti-correlation with the rain season is proved.

\section{Acknowledgement}

This work was supported in Russia by RFBR (grants 14-02-00996 and 13-02-00574), RAS Presidium Program "Fundamental properties of matter and astrophysics", and in China by NSFC (No.10975046, No.11375052). We also acknowledge the support of the ARGO-YBJ collaboration.

\section{References}

[1] Alekseenko, V. V. et al. Correlation of variations in the thermal neutron flux from the earth's crust with the moon's phases and with seismic activity. Izvestiya, Physics of the Solid Earth, 45, No. 8, 709-718 (2009).

[2] Alekseenko, V.V., Gavrlyuk, Yu.M., Kuzminov, V.V. and Stenkin, Yu.V.. Tidal effect in the radondue neutron flux from the Earth's crust. J. of Physics: Conference Series. 203, 012045 (2010)

[3] Sten'kin, Yuri V. Large scintillator detector for thermal neutron recording. Editor: Sidorov, Maksim \& Ivanov, Oleg, Nuclear Track Detectors: Design, Methods and Applications, Chapter 10, 253-256 (ISBN: 978-1-60876-826-4, Nova Science Publishers, Inc., 2010).

[4] Ma, X. H. for ARGO-YBJ \& Stenkin, Yuri for PRISMA-YBJ. Coincident air shower events between ARGO-YBJ and PRISMA-YBJ. the 33d ICRC proceeding, Rio de Janeiro, ID 606 (2013).

[5] D'Ettorre, Piazzoli Benedetto on behalf of the ARGO-YBJ collaboration, Highlights from the ARGO-YBJ experiment, The 32nd ICRC proceeding, Beijing, 12, 93-106 (2011).

[6] Cigolini, C. et al. Radon surveys and real-time monitoring at Stromboli volcano: Influence of soil temperature, atmospheric pressure and tidal forces on ${ }^{222} \mathrm{Rn}$ degassing. Journal of Volcanology and Geothermal Research 184, 381-388(2009).

[7] Zmazek, B. et al. Application of decision trees to the analysis of soil radon data for earthquake prediction. Applied Radiation and Isotopes, 58, 697-706 (2003).

[8] Firstov, P. P. \& Rudakov, V. P. Results of recording of subsurface radon in 1997-2000 at the Petropavlovsk Kamchatski geodynamic research area." Vulkanologiya i Seismologiya, 1, 2641(2003). 


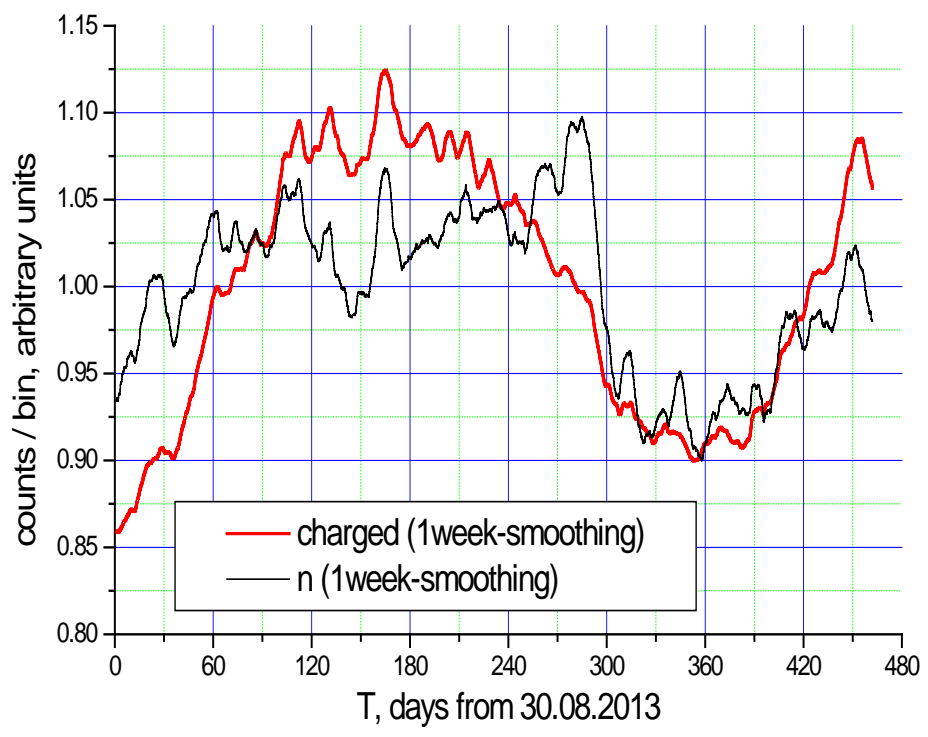

Figure 1: Seasonal effects for different parameters. "neutrons" and "charged" channels with 1-week smoothing

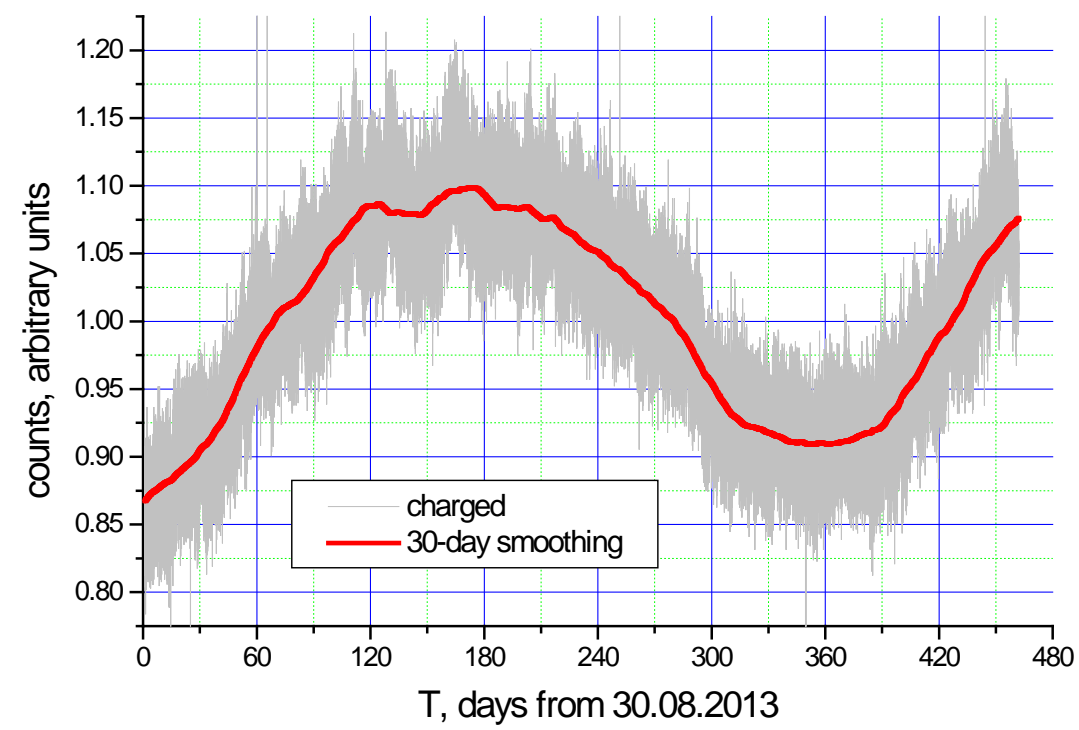

Figure 2: Seasonal effects for "charged" channel with and without 1-month smoothing 


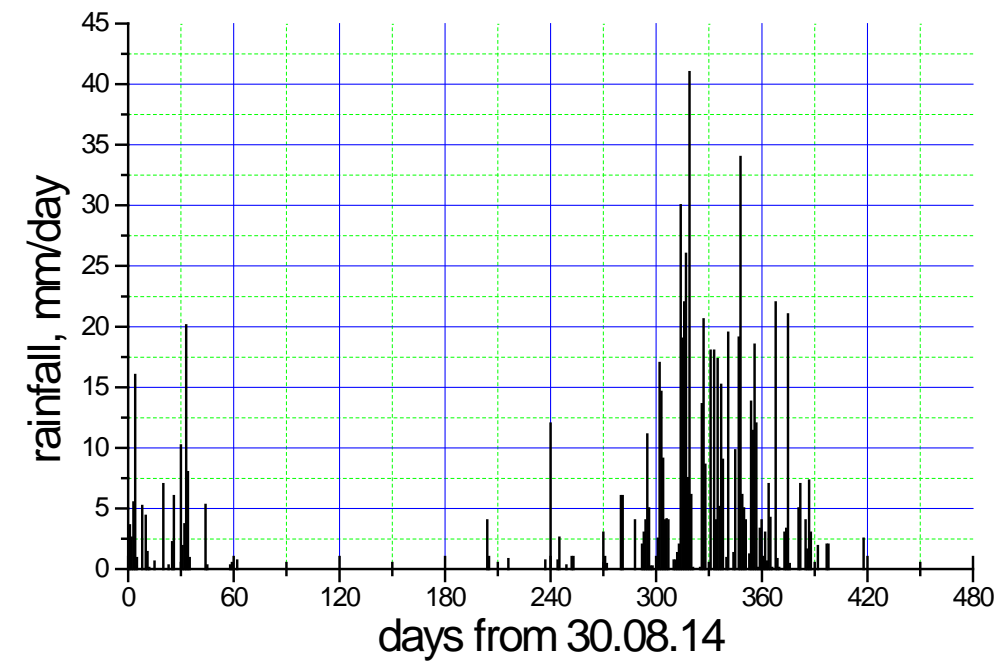

Figure 3: Rainfall in mm/day (taken from http://rp5.ru).

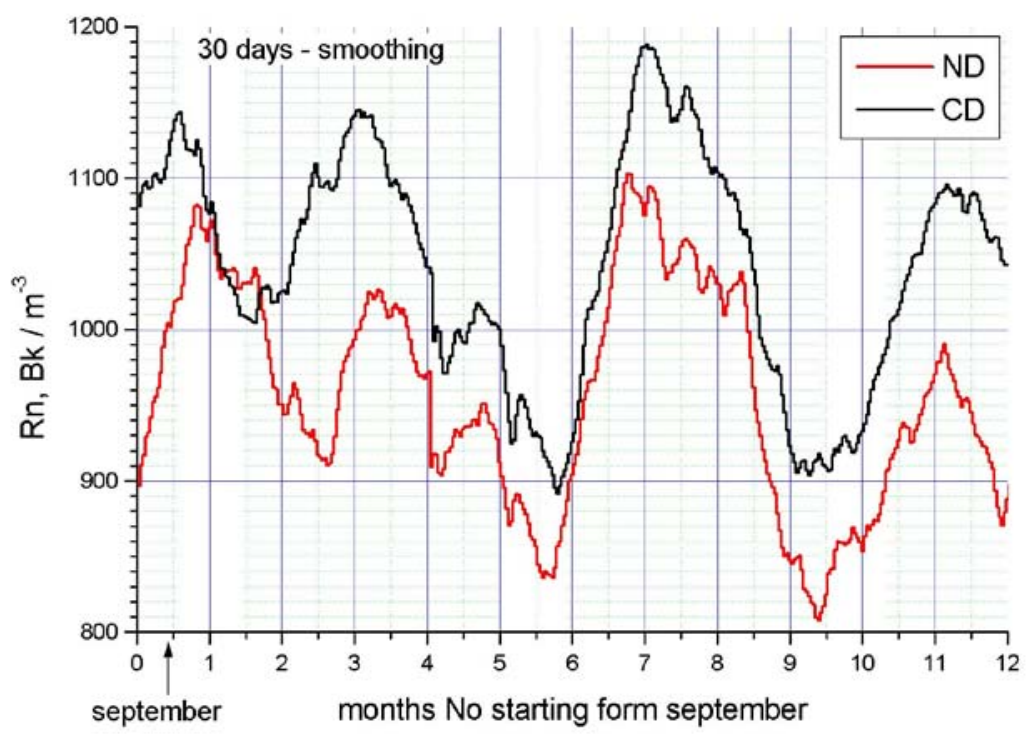

Figure 4: air radon concentration measured by radon meters (Lukas cells) in two points of the ARGO-YBJ hall (ND - north and CD - central detectors) from Sep. till Aug. and averaged over 2 years. 